\title{
Role of elevated liver transaminase levels in the diagnosis of liver injury after blunt abdominal trauma
}

\author{
ZHIQIANG TIAN*, HONG LIU* ${ }^{*}$ XIAOFANG SU, ZHENG FANG, \\ ZHITAO DONG, CHANGCHUN YU and KUNLUN LUO \\ Department of Hepatobiliary Surgery, 101st Hospital of the Chinese People's \\ Liberation Army, Wuxi, Jiangsu, P.R. China
}

Received March 1, 2012; Accepted May 3, 2012

DOI: $10.3892 /$ etm.2012.575

\begin{abstract}
The liver is the second most commonly injured organ following blunt abdominal trauma. The stable patient with minimal physical findings with a history of blunt abdominal trauma presents a challenge for diagnosis of liver injury. This study was conducted to determine the usefulness of hepatic transaminases in predicting the presence of liver injury and its severity following blunt abdominal trauma. In this retrospective study, we included all patients who had sustained blunt abdominal injury and were treated at our institution between January 2008 and December 2010. The grading of the liver injury was verified using CT scans or surgical findings. One hundred and eighty-two patients with blunt abdominal trauma underwent the required blood tests and were included in the study. Using receiver operating characteristic (ROC) curve assessment, optimum alanine aminotransferase (ALT), aspartate aminotransferase (AST), lactate dehydrogenase (LDH) and $\gamma$-glutamyl transpeptidase (GGT) thresholds were determined to be $>57 \mathrm{U} / 1,113 \mathrm{U} / 1,595 \mathrm{U} / 1$ and $50 \mathrm{U} / 1$. ALT $>57 \mathrm{U} / 1$ $(\mathrm{OR}, 66.1 ; \mathrm{P}<0.001)$ and $\mathrm{AST}>113 \mathrm{U} / 1(\mathrm{OR}, 30.6 ; \mathrm{P}<0.001)$ were strongly associated with the presence of liver injuries. This association was also observed in patients with elevated LDH >595 U/1 (OR, 3.8; P<0.001) and GGT >50 U/1 (OR, $3.0 ; \mathrm{P}<0.05)$. None of the laboratory tests were related to the severity of the liver injuries. In patients with blunt abdominal trauma, abnormal hepatic transaminase levels are associated with liver injuries. Patients with ALT >57 U/1 and AST $>113 \mathrm{U} / \mathrm{l}$ are strongly associated with liver injury and require further imaging studies and close management.
\end{abstract}

Correspondence to: Dr Kunlun Luo, Department of Hepatobiliary Surgery, 101st Hospital of Chinese People's Liberation Army, Wuxi, Jiangsu, P.R. China

E-mail: zhiqiangtiann@gmail.com

*Contributed equally

Key words: liver injury, blunt trauma, transaminase, computed tomography, retrospective study

\section{Introduction}

The liver is the second most commonly injured organ following blunt abdominal trauma and associated injuries contribute significantly to mortality and morbidity (1). Early diagnosis of the nature and extent of intra-abdominal organ injuries may result in significant reduction of morbidity and mortality (1). Focused abdominal sonography for trauma (FAST) is able to sensitively detect free fluid in the abdomen and pelvis, but its numerous limitations have been recognized (2-4). The overall sensitivity of emergency FAST for detection of blunt liver injury was reported to be as low as $64 \%$ (5). When there are parenchymal injuries of the liver only, with no free fluid, the sensitivity is even lower (6). Computed tomography (CT) is the standard diagnostic modality for stable trauma patients with a suspected abdominal injury $(7,8)$. However, accurate diagnosis of significant injuries could be delayed as not all health institutions worldwide have ready access to CT scans. In addition, a CT scan suite, at times, may not provide a safe environment for resuscitation and additionally has limitations for patients who are too unstable for transportation. The high cost of a CT scan does not permit its widespread use in screening all patients with blunt abdominal trauma.

In view of the above issues, small-scale studies carried out in pediatric patients with trauma have revealed varied threshold admission levels of liver enzymes below which no clinically significant liver injury was observed (9-13). Should an association between laboratory tests and liver injuries exist, early identification of patients with liver injuries could be achieved. The usefulness of these tests in predicting intraabdominal and liver injury in adults has not been intensively investigated. The present study was undertaken to determine the accuracy of selected laboratory tests in predicting the presence of liver injury and its severity following blunt abdominal trauma.

\section{Materials and methods}

Study population. The 101st Hospital of the Chinese People's Liberation Army is a 1,000-bed hospital in Southeastern China that provides medical care to approximately 1 million individuals within the Wuxi metropolitan area and has more than 10,000 visits to the emergency department annually. It admits 
an average of 600 serious trauma cases yearly, of which $96 \%$ are blunt injuries.

A review of a retrospective electronic database of all patients with traumatic blunt abdominal injuries to our institution over a three-year period (January 2008 to December 2010) was performed. Patients were excluded if they suffered penetrating injuries, died in the emergency department or if the required laboratory tests were not performed within $24 \mathrm{~h}$ of the trauma.

Patients were then subdivided into two groups: patients with and without liver injuries. Liver injury grade was determined using the organ injury scale (1994 revision) described by the American Association of Surgery for Trauma (AAST). In this study, minor liver injuries were classified as AAST Grades I-III, while major liver injuries were classified as AAST Grades IV-VI. The information was obtained from either surgery or CT scans. The results of the CT scans were abstracted from attending radiology reports if the CT scan was performed at our hospital or from review of progress notes if the scan was obtained at another institution prior to transfer.

Test results for aspartate aminotransferase (AST), alanine aminotransferase (ALT), alkaline phosphatase (ALP), lactate dehydrogenase (LDH), bilirubin, $\gamma$-glutamyl transpeptidase (GGT), C-reactive protein (CRP) and white blood cell (WBC) levels were collected. The values were compared with reference ranges for our institution (Table I). Data collection forms also included age, gender, trauma mechanisms, injury severity score (ISS), the AAST grade of liver injury, length of stay $(\mathrm{LoS})$ in an intensive care unit (ICU), total inpatient LoS and the eventual outcome.

Median and range were calculated for continuous variables. The selected laboratory test levels in patients with liver injuries were compared with patients with abdominal non-liver injury by plotting the receiver operating characteristic (ROC) curves for threshold in the presence or absence of liver injury. Data analysis was performed by comparing the selected laboratory tests to the threshold using the Chi-square test. All P-values were 2 -sided and considered significant at $\mathrm{P}<0.05$. All statistical operations were performed using SPSS Statistics 13 for Windows (SPSS Inc, Chicago, IL, USA).

\section{Results}

Characteristics of the study sample. During the three-year study period, 182 patients with blunt abdominal trauma had the relevant laboratory tests performed and were included in our study. Table II summarizes the general characteristics of the two groups. In the two groups there was a preponderance of males. Patients with abdominal non-liver injury were, on average, older than those with liver injury. The majority of the patients underwent $\mathrm{CT}$ scans. The road traffic accident was the most common mechanism of injury. Patients with liver injury tended to be significantly more severely injured than those with non-liver injury in terms of their median ISS, LoS in ICU and total. However, more patients $(67.4 \%)$ with abdominal non-liver injury underwent exploratory laparotomy than those (41.1\%) with liver injury.

Patients with liver injuries. The grading of the 90 patients with liver injuries is shown in Table III. In those patients with liver injuries, patients were graded according to the severity
Table I. Reference ranges for laboratory tests.
Laboratory test

Aspartate aminotransferase (AST)

Alanine aminotransferase (ALT)

Alkaline phosphatase (ALP)

Bilirubin

Lactate dehydrogenase (LDH)

$\gamma$-glutamyl transpeptidase (GGT)

White blood cell (WBC)

C-reactive protein (CRP)
Reference range

$0-50 \mathrm{U} / 1$

$0-50 \mathrm{U} / 1$

$32-135 \mathrm{U} / 1$

4-25 $\mu \mathrm{mol} / 1$

$50-250 \mathrm{U} / 1$

$0-50 \mathrm{U} / 1$

$4-10 \times 10^{9} / 1$

$0-5 \mathrm{mg} / \mathrm{l}$ of their liver injury as follows: grade I, 23 patients; grade II, 27 patients; grade III, 23 patients; grade IV, 6 patients; and grade V, 11 patients. There were no patients with grade VI liver injuries. Grouped according to severity, there were 73 patients $(81.1 \%)$ with minor (grades I-III) injuries and 17 patients $(18.9 \%)$ with major (grades IV-V) injuries.

Main results. Patients with non-liver injury and grades of liver injury from I to $\mathrm{V}$ were significantly different in regards to levels of ALT $(\mathrm{P}<0.001)$, AST $(\mathrm{P}<0.001)$, LDH $(\mathrm{P}<0.001)$ and GGT $(\mathrm{P}=0.001)$ (Table IV). Fig. 1 shows the ROC curves generated for the sensitivity and specificity comparison for the association between ALT, AST, LDH, GGT levels and the presence of liver injury. The area under the curve demonstrated that the test was a good discriminator for identification of liver injury. Using ROC curve assessment, optimum ALT, AST, LDH, GGT thresholds were determined to be $>57 \mathrm{U} / 1,113 \mathrm{U} / 1,595 \mathrm{U} / 1$ and $50 \mathrm{U} / 1$.

ALT $>57 \mathrm{U} / 1(\mathrm{OR}, 66.1 ; \mathrm{P}<0.001)$ and AST >113 U/1 (OR, 30.6 ; $\mathrm{P}<0.001)$ were significantly associated with the presence of liver injuries. This was also observed in patients with $\mathrm{LDH}>595 \mathrm{U} / 1(\mathrm{OR}, 3.8 ; \mathrm{P}<0.001)$ and GGT > 50 U/1 (OR, 3.0; $\mathrm{P}<0.05)$ (Table V).

Further analysis of selected laboratory tests also revealed that ALT $>57 \mathrm{U} / 1$ is perhaps most suitable for detecting hepatic injuries. Its sensitivity (92.2\%), specificity (84.8\%), positive predictive value $(85.6 \%)$ and negative predictive value $(91.8 \%)$ are all favorable for its role as a screening tool compared to the other markers (Table VI). However, in patients with liver injuries, none of the selected laboratory tests were related to the severity of the liver injuries (Table VII).

\section{Discussion}

The liver continues to be the second most commonly injured organ in blunt abdominal trauma (1). Physicians dealing with blunt abdominal trauma often use biochemical tests and radiographic imaging to aid in clinical assessment. Outcome of liver trauma has been shown to be related to several important factors: increased ISS, worse grading of hepatic injury, advanced age, operative blood loss and hemodynamic instability on admission $(14,15)$.

Patients with blunt abdominal trauma resulting in liver injury may present with unstable hemodynamics and obvious hemoperitoneum. These patients usually do not represent a diagnostic challenge since the strategy is clear. They gener- 
Table II. Characteristics of the 182 patients with blunt abdominal trauma.

\begin{tabular}{|c|c|c|}
\hline & $\begin{array}{l}\text { Liver injury } \\
\qquad(\mathrm{n}=90)\end{array}$ & $\begin{array}{l}\text { Abdominal non-liver } \\
\text { injury }(n=92)\end{array}$ \\
\hline Age, median (range) & $39.0(8-73)$ & $45.7(10-83)$ \\
\hline \multicolumn{3}{|l|}{ Gender, n (\%) } \\
\hline Male & $75(83.3)$ & $72(78.3)$ \\
\hline Female & $15(16.7)$ & $20(21.7)$ \\
\hline \multicolumn{3}{|l|}{ Mechanism, n (\%) } \\
\hline Road traffic accident & $57(63.3)$ & $58(63.0)$ \\
\hline Fall & $21(23.3)$ & $16(17.4)$ \\
\hline Assault & 7 (7.8) & $12(13.0)$ \\
\hline Crush & $5 \quad(5.6)$ & $6 \quad(6.5)$ \\
\hline \multicolumn{3}{|l|}{ CT scans, n (\%) } \\
\hline Performed & 89 (98.9) & $84(91.3)$ \\
\hline Not performed & 1 (1.1) & $8(8.7)$ \\
\hline \multicolumn{3}{|l|}{ Surgical intervention, $\mathrm{n}(\%)$} \\
\hline Performed & $37(41.1)$ & $62(67.4)$ \\
\hline Not performed & $53(58.9)$ & $30(32.6)$ \\
\hline ISS, median (range) & $32.0(9-75)$ & $24.6(9-75)$ \\
\hline ICU LoS, median (range) & $6.9(0-30)$ & $5.0(1-30)$ \\
\hline Total LoS, median (range) & $30.3(2-215)$ & $22.1(2-93)$ \\
\hline \multicolumn{3}{|l|}{ Outcome, n (\%) } \\
\hline Alive & $85(94.4)$ & $83(90.2)$ \\
\hline Dead & $5(5.6)$ & $9(9.8)$ \\
\hline
\end{tabular}

CT, computed tomography; ISS, injury severity score; ICU, intensive care unit; LoS, length of stay.
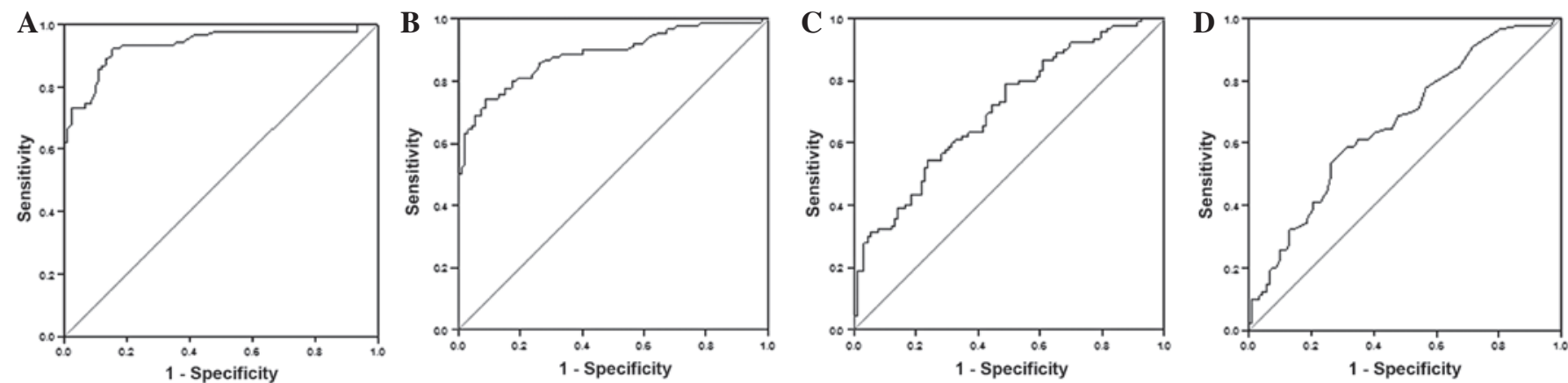

Figure 1 ROC curves showing sensitivity and specificity for (A) alanine aminotransferase, (B) aspartate aminotransferase, (C) lactate dehydrogenase and (D) $\gamma$-glutamyl transpeptidase threshold values. ROC, receiver operating characteristic. Diagonal segments are produced by ties.

Table III. Grading of liver injury for the 90 patients.

\begin{tabular}{lr}
\hline Grading of liver injury & $\mathrm{n}(\%)$ \\
\hline Minor liver injuries & $73(81.1)$ \\
I & $23(25.6)$ \\
II & $27(30.0)$ \\
III & $23(25.6)$ \\
Major liver injuries & $17(18.9)$ \\
IV & $6(6.7)$ \\
V & $11(12.2)$ \\
VI & $0(0.0)$ \\
\hline
\end{tabular}

ally receive either prompt abdominal imaging (ultrasound or CT scan) or laparotomy or both. Usually the more difficult diagnosis is that of lesser, but still significant, liver injury in the stable patient with minimal physical findings following blunt abdominal trauma. Abdominal CT scan has proved to be a valuable diagnostic tool for evaluating intra-abdominal injury (8). However, it may only be carried out if available and in patients who are hemodynamically stable. Furthermore, it is costly, requires radiation exposure, and removes patients from direct clinical care. Another useful tool for initial survey is FAST. Yet, FAST is not always available in all healthcare institutions and one of the major limitations is the technical 
Table IV. Relationship between the various laboratory tests and liver injuries.

\begin{tabular}{|c|c|c|c|c|c|c|c|c|}
\hline \multirow{2}{*}{$\begin{array}{l}\text { Laboratory } \\
\text { test }\end{array}$} & \multirow[b]{2}{*}{ No liver injury } & \multicolumn{5}{|c|}{ Grade of liver injury } & \multirow[b]{2}{*}{ F-value } & \multirow[b]{2}{*}{ P-value } \\
\hline & & I & II & III & IV & V & & \\
\hline ALT & $35 \pm 23$ & $96 \pm 64$ & $306 \pm 274$ & $263 \pm 206$ & $628 \pm 448$ & $681 \pm 440$ & 39.01 & $<0.001$ \\
\hline AST & $59 \pm 42$ & $126 \pm 90$ & $309 \pm 261$ & $292 \pm 230$ & $777 \pm 482$ & $695 \pm 368$ & 43.40 & $<0.001$ \\
\hline LDH & $483 \pm 379$ & $637 \pm 509$ & $879 \pm 1084$ & $775 \pm 652$ & $2023 \pm 1715$ & $1581 \pm 1011$ & 10.27 & $<0.001$ \\
\hline ALP & $57 \pm 39$ & $57 \pm 36$ & $65 \pm 27$ & $60 \pm 28$ & $52 \pm 15$ & $60 \pm 31$ & 0.28 & 0.923 \\
\hline GGT & $25 \pm 37$ & $28 \pm 35$ & $83 \pm 138$ & $32 \pm 36$ & $73 \pm 62$ & $29 \pm 14$ & 4.12 & 0.001 \\
\hline Bilirubin & $17 \pm 10$ & $18 \pm 13$ & $17 \pm 11$ & $14 \pm 7$ & $18 \pm 6$ & $25 \pm 24$ & 1.20 & 0.310 \\
\hline WBC & $14 \pm 6$ & $13 \pm 7$ & $14 \pm 6$ & $13 \pm 5$ & $17 \pm 6$ & $11 \pm 2$ & 0.97 & 0.439 \\
\hline CRP & $54 \pm 80$ & $52 \pm 84$ & $53 \pm 87$ & $31 \pm 32$ & $16 \pm 29$ & $40 \pm 67$ & 0.63 & 0.681 \\
\hline
\end{tabular}

ALT, alanine aminotransferase; AST, aspartate aminotransferase; LDH, lactate dehydrogenase; ALP, alkaline phosphatase; GGT, $\gamma$-glutamyl transpeptidase; WBC, white blood cell count; CRP, C-reactive protein.

Table V. Relationship between selected laboratory tests and the presence of liver injuries (part I).

\begin{tabular}{|c|c|c|c|c|c|}
\hline Laboratory test & Liver injury (n) & No liver injury (n) & OR & $95 \% \mathrm{CI}$ & P-value \\
\hline Median ALT (range) & $309(13-1500)$ & $35(8-118)$ & 66.1 & $25.33-172.27$ & $<0.001$ \\
\hline $\mathrm{ALT} \leq 57$ & 7 & 78 & & & \\
\hline $\mathrm{ALT}>57$ & 83 & 14 & & & \\
\hline Median AST (range) & $336(17-1637)$ & $59(15-228)$ & 30.6 & $12.86-72.73$ & $<0.001$ \\
\hline $\mathrm{AST} \leq 113$ & 23 & 84 & & & \\
\hline AST $>113$ & 67 & 8 & & & \\
\hline Median LDH (range) & $953(173-5787)$ & $482(49-2875)$ & 3.8 & $2.02-7.17$ & $<0.001$ \\
\hline LDH $\leq 595$ & 41 & 70 & & & \\
\hline $\mathrm{LDH}>595$ & 49 & 22 & & & \\
\hline Median GGT (range) & $49(4-551)$ & $25(3-319)$ & 3.0 & $1.25-7.22$ & 0.014 \\
\hline $\mathrm{GGT} \leq 50$ & 70 & 84 & & & \\
\hline GGT $>50$ & 20 & 8 & & & \\
\hline
\end{tabular}

ALT, alanine aminotransferase; AST, aspartate aminotransferase; LDH, lactate dehydrogenase; ALP, alkaline phosphatase; GGT, $\gamma$-glutamyl transpeptidase; OR, odds ratio; CI, confidence interval.

expertise of the operator $(3,4)$. Furthermore, FAST has limited value if the blunt abdominal trauma does not result in hemoperitoneum, and it may miss nearly one third of the cases of abdominal injuries when used as the sole diagnostic tool in evaluating victims of blunt abdominal trauma.

Elevation of the serum liver enzymes AST and ALT is known to be associated with blunt traumatic liver injury. Presumably, as these transaminases are present in high concentrations in hepatocytes, they are released into the circulation in large quantities following acute traumatic hepatocellular injury. AST and ALT have been previously reported to indicate liver injury $(12,16)$. One previous observational cohort study has reported serum ALT to be a sensitive diagnostic marker when evaluating harm caused by blunt hepatic injuries (17).

From our study, the authors preferred ALT to AST as a screening tool for hepatic injuries due to its associated high sensitivity, specificity, positive predictive value and negative predictive value. Although 7 (7.8\%) and $23(25.6 \%)$ of the patients with liver injuries had ALT and AST levels less than their thresholds in our study, most of them only had grades I and II injuries. We also found a trend that the more severe the liver injury of the patients, the higher the liver enzyme levels, but our study further demonstrates that patients with elevated ALT >100 U/1, AST >113 U/1, LDH >595 U/1 and GGT >100 U/1 did not necessarily have major liver injury. Therefore, even where patients whose liver enzyme levels are at lower levels and liver injury cannot be completely ruled out, they may still provide clues concerning liver injury, particularly in patients with high-grade liver injury.

There are several limitations to this study. Firstly, it is a retrospective chart review; therefore, data may not be present or properly recorded on the medical record. Secondly, the time interval between injury and the procurement of the blood test could not be standardized. This was predominantly due to the highly variable timing and location of the accident. However, 
Table VI. Relationship between selected laboratory tests to presence of liver injuries (part II).

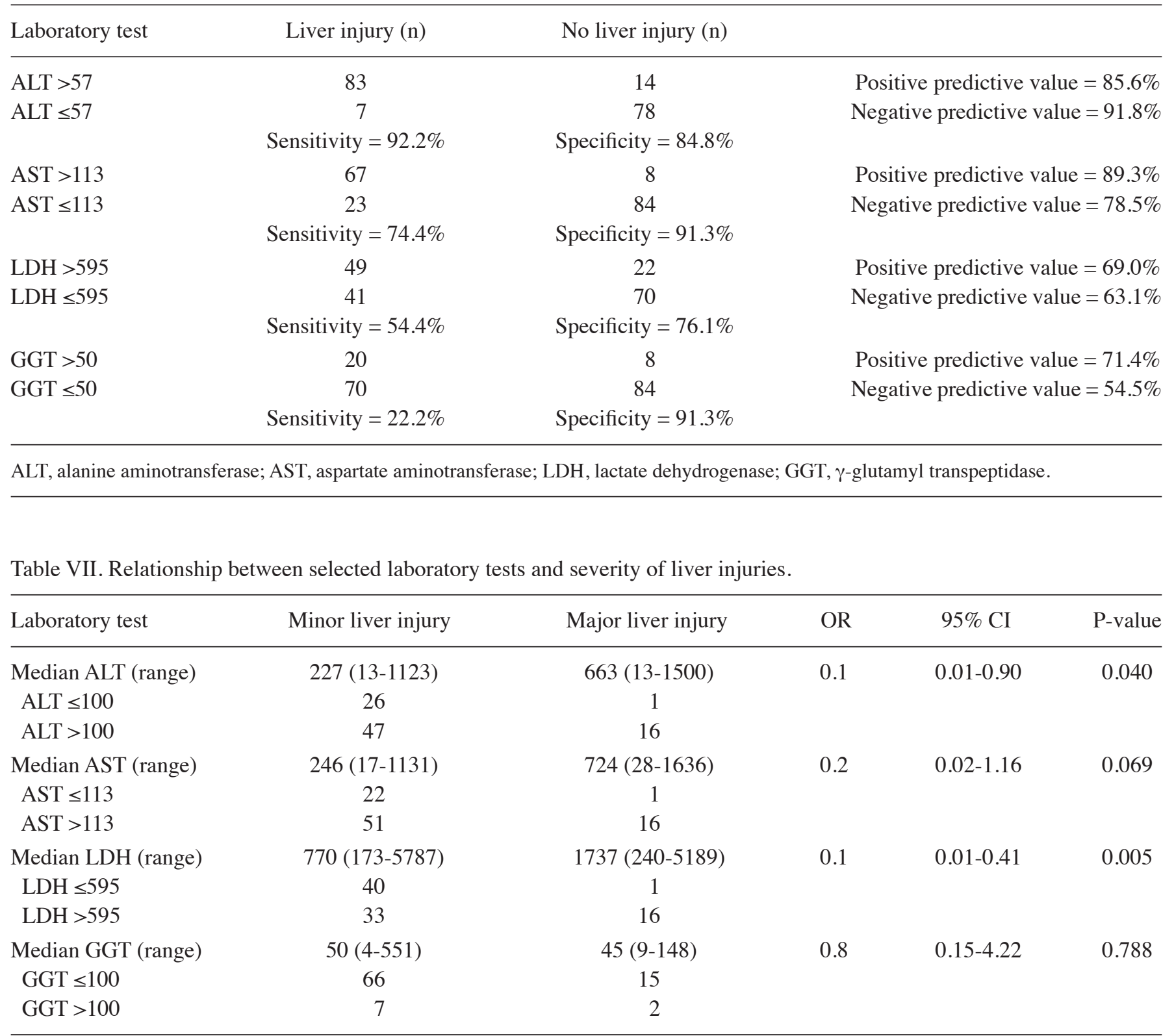

ALT, alanine aminotransferase; AST, aspartate aminotransferase; LDH, lactate dehydrogenase; ALP, alkaline phosphatase; GGT, $\gamma$-glutamyl transpeptidase; OR, odds ratio; CI, confidence interval.

we tried to confine our patients to the group having their blood drawn within $24 \mathrm{~h}$ to reduce study bias. Thirdly, as the threshold for undertaking CT scans varies greatly from one institution to another, our findings may not be applicable to the entire population of patients with blunt abdominal trauma.

In conclusion, this study suggests that, in patients with blunt abdominal trauma, abnormal transaminase levels are associated with liver injury. Patients with ALT $>57 \mathrm{U} / 1$ and AST $>113 \mathrm{U} / 1$ are strongly associated with liver injury and require further imaging studies and close management.

\section{Acknowledgements}

This research was supported by the Army Medicine Technology Fund (10MA008).

\section{References}

1. Piper GL and Peitzman AB: Current management of hepatic trauma. Surg Clin North Am 90: 775, 2010.

2. Nural MS, Yardan T, Guven H, et al: Diagnostic value of ultrasonography in the evaluation of blunt abdominal trauma. Diagn Interv Radiol 11: 41-44, 2005.

3. Scalea TM, Rodriguez A, Chiu WC, et al: Focused assessment with sonography for trauma (FAST): results from an international consensus conference. J Trauma 46: 466-472, 1999.

4. Chiu WC, Cushing BM, Rodriguez A, et al: Abdominal injuries without hemoperitoneum: a potential limitation of focused abdominal sonography for trauma (FAST). J Trauma 42: 617-623, 1997.

5. Kirkpatrick AW, Sirois M, Laupland KB, et al: Prospective evaluation of hand-held focused abdominal sonography for trauma (FAST) in blunt abdominal trauma. Can J Surg 48: 453-460, 2005.

6. Richards JR, McGahan JP, Pali MJ, et al: Sonographic detection of blunt hepatic trauma: hemoperitoneum and parenchymal patterns of injury. J Trauma 47: 1092-1097, 1999. 
7. Richards JR and Derlet RW: Computed tomography for blunt abdominal trauma in the ED: a prospective study. Am J Emerg Med 16: 338-342, 1999.

8. Kreimeyer S and Grenacher L: Modern imaging techniques for liver trauma. Chirurg 80: 896, 2009 (In German).

9. Sola JE, Cheung MC, Yang R, et al: Pediatric FAST and elevated liver transaminases: an effective screening tool in blunt abdominal trauma. J Surg Res 157: 103-107, 2009.

10. Capraro AJ, Mooney D and Waltzman ML: The use of routine laboratory studies as screening tools in pediatric abdominal trauma. Pediatr Emerg Care 22: 480-484, 2006.

11. Karaduman D, Sarioglu-Buke A, Kilic I, et al: The role of elevated liver transaminase levels in children with blunt abdominal trauma. Injury 34: 249-252, 2003.

12. Puranik SR, Hayes JS, Long J, et al: Liver enzymes as predictors of liver damage due to blunt abdominal trauma in children. South Med J 95: 203-206, 2002.
13. Karam O, La Scala G, Le Coultre C, et al: Liver function tests in children with blunt abdominal traumas. Eur J Pediatr Surg 17: 313-316, 2007.

14. Scollay JM, Beard D, Smith R, et al: Eleven years of liver trauma: the Scottish experience. World J Surg 29: 744-749, 2005.

15. Sikhondze WL, Madiba TE, Naidoo NM, et al: Predictors of outcome in patients requiring surgery for liver trauma. Injury 38 : 65-70, 2007.

16. Stassen NA, Lukan JK, Carrillo EH, et al: Examination of the role of abdominal computed tomography in the evaluation of victims of trauma with increased aspartate aminotransferase in the era of focused abdominal sonography for trauma. Surgery 132: 642-646, 2002.

17. Srivastava AR, Kumar S, Agarwal GG, et al: Blunt abdominal injury: serum ALT-A marker of liver injury and a guide to assessment of its severity. Injury 38: 1069-1074, 2002. 